\title{
IDEMPOTENTS IN GROUP RINGS
}

\author{
D. B. COLEMAN
}

The following result generalizes a corollary of a theorem due to Swan $\left[1\right.$, p. 571]. ${ }^{1}$ Swan's theorem deals with Dedekind rings of characteristic 0 .

TheOREM. Let $R$ be an integral domain (with 1 ), and let $G$ be a finite group of order $n$. Then the group ring $R G$ has an idempotent different from 0 or 1 if and only if some prime divisor of $n$ is a unit in $R$.

Proof. Suppose that $p$ is a prime dividing $n$, and that $p$ is a unit in $R$. Let $P$ be a subgroup of $G$ of order $p$, and let $e=p^{-1} \sum_{x \in P} x$. Then $e$ is a nontrivial idempotent.

Conversely, suppose that $e=\sum_{g \in G} \alpha_{g} g$ is a nontrivial idempotent in $R G$ and that the characteristic of $R$ is zero. Let $E=\left(e_{i j}\right)$ be the matrix representing $e$ under the regular representation. Then $e_{i i}=\alpha_{1}$ for $i=1, \cdots, n$, so the trace of $E$ is $n \alpha_{1}=q$, the multiplicity of 1 as an eigenvalue of $E$. Since $e \neq 0,1$, we have that $0<q<n$. Let $n_{1}$ and $q_{1}$ be the relatively prime integers such that $n_{1}$ divides $n, q_{1}$ divides $q$, and $n_{1} \alpha_{1}=q_{1}$. Let $a$ and $b$ be integers such that $a n_{1}+b q_{1}=1$; then $\left(a+b \alpha_{1}\right) n_{1}=1$, so that $n_{1}$ is a unit in $R$. Since $q<n$, we see that $n_{1} \neq 1$. Any prime divisor of $n_{1}$ is a unit in $R$.

Next, let $R$ have characteristic $p \neq 0$, and let $n=p^{r} t$, where $(p, t)=1$ and $r \geqq 0$.

It is well known that in the group algebra of a $p$-group over a field of characteristic $p$, every zero divisor is nilpotent. In particular, this is the case in $K G$ if $t=1$, where $K$ denotes the quotient field of $R$.

Suppose that $R G$ has a nontrivial idempotent $e$. Then $e$ is a nonnilpotent zero divisor in $R G$ (hence in $K G$ ). Thus by the above, $t \neq 1$. Clearly $t$ is a unit in $R$.

\section{BIBLIOGRAPHY}

1. R. G. Swan, Induced representations and projective modules, Ann. of Math. 71 (1960), 552-578.

VANDERBilt UNiversity

Received by the editors March 14, 1966.

1 The author is indebted to the referee for this reference. 